Address for Correspondence: Dr. Zhi-Yong Peng,

Department of Critical Care Medicine, Zhongnan Hospital, Wuhan University School of Medicine, Wuhan 430071, Hubei Province, China or

Center of Critical Care Nephrology, Department of Critical

Care Medicine, University of Pittsburgh

School of Medicine, Pittsburgh, PA

15213, USA.

Email: pengzy5@hotmail.com

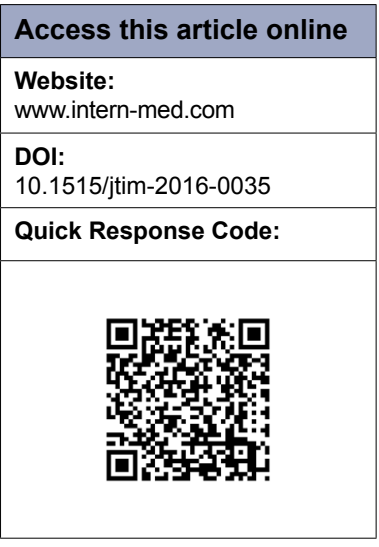

\title{
The biomarkers for acute kidney injury: A clear road ahead?
}

\author{
Zhi-Yong Peng ${ }^{1,2}$ \\ 'Department of Critical Care Medicine, Zhongnan Hospital, Wuhan University School of Medicine, Wuhan \\ 430071, Hubei Province, China; \\ ${ }^{2}$ Center of Critical Care Nephrology, Department of Critical Care Medicine, University of Pittsburgh School of \\ Medicine, Pittsburgh, PA 15213, USA
}

Acute kidney injury (AKI) is a common and serious complication that is associated with several adverse outcomes in hospitalized patients. Serum creatinine is widely used in diagnosing the presence of AKI, but it is a lagging marker of change in kidney function, with poor sensitivity. Thus, different urinary and serum proteins have been intensively investigated as possible biomarkers for early diagnosis of AKI. $\mathrm{AKI}$ is a complex process, and biomarkers could be used for different roles at different stages. In addition to facilitating early diagnosis, AKI biomarkers can provide valuable insight into risk stratification for the vulnerable patients. Furthermore, biomarkers could also function as molecular phenotyping tools that could be used to guide clinical intervention. However, the effective use of biomarkers requires an understanding of their strengths, limitations and individual performance characteristics. This manuscript will review the role of a variety of biomarkers in AKI management, and discuss how they can be used in current clinical practice.

\section{THE ROLE OF BIOMARKERS IN AKI RISK STRATIFICATION}

Susceptibility to AKI is the result of multiple interactions and risk factors. Due to lack of effective treatments for AKI, supportive care is the mainstay of therapy and prevention of AKI is paramount. Understanding an individual's AKI risk profile may offer the opportunity for prevention or early intervention. Patients with chronic liver disease/hepatic failure, hypertension, diabetes, and advanced age are at high risk of AKI if exposed to sepsis, nephrotoxins or cardiopulmonary bypass. Effective risk stratification to identify these patients is essential.

Several AKI risk prediction scores and kidney-specific scoring models have been developed and validated in the setting of cardiac surgery; however most of these scores fail to predict milder forms of AKI. The currently available risk scores used to predict AKI are often not sensitive or specific enough to identify high-risk individuals, and poorly predict the AKI progression. Recently, the risk stratification of AKI has been evaluated and refined by the use of functional and damage biomarkers. These candidate biomarkers were identified based on the AKI pathophysiology. Creatinine is a functional marker, a surrogate for changes in the glomerular filtration rate, which often occurs late in the course of AKI. Injury biomarkers can detect AKI up to 12 to 66 hours before increases in serum creatinine, and predict those patients who are most likely to develop AKI even before an inciting event. Subclinical AKI is marked with a lack of change in serum creatinine, but with changes in structural nephron damage biomarkers.

Cardiac surgery is one of the main risk factors for AKI, due to the ischemic kidney insult during cardiopulmonary bypass..$^{[1,2]}$ Both urinary and serum cystatin $C$ have been studied in the Translational Research Investigating Biomarker Endpoints in AKI (TRIBE-AKI) cohort, one of the largest international studies in patients undergoing cardiac surgery. The preoperative serum 
cystatin $\mathrm{C}$ performed better than serum creatinine at predicting the risk of AKI, but the urinary cystatin $\mathrm{C}$ did not yield similar results.

Risk stratification of $\mathrm{AKI}^{[3]}$ is important for acute decompensated heart failure (ADHF). For predicting intrinsic AKI on the first day of intensive care unit (ICU) admission, a combination of serum cystatin- $C$ and urine kidney injury molecule-1 (KIM-1) yielded an excellent area under the receiver operating characteristic curve, with high sensitivity and specificity. In patients with ST-segment elevation myocardial infarction (STEMI), undergoing primary percutaneous coronary intervention, urinary neutrophil gelatinase-associated lipocalin (NGAL) and cystatin $\mathrm{C}$ at presentation were predictive of AKI. In addition, high serum levels of these biomarkers were associated with an elevated risk and a more advanced stage of AKI. Combining urinary NGAL with a novel creatininebased metric, both available soon after the completion of surgery, may provide previously unavailable early and effective risk stratification for serious adverse outcomes after cardiac surgery.

Urinary insulin-like growth factor binding protein (IGFBP) 7 and tissue inhibitor of metalloproteinase (TIMP)- 2 were found to be increased in the urine of patients at high risk of AKI from a variety of etiologies..$^{[4]}$ These markers could be useful in clinical practice to detect subclinical episodes of AKI, or to make an early identification of patients at risk. In 2014, the US Food and Drug Administration approved the marketing of a test based on the combination of urine concentrations of [TIMP-2] [IGFBP7] (the product of TIMP-2 and IGFBP7 levels) to determine whether critically ill patients are at risk for developing moderate to severe AKI. Urinary [TIMP-2] [IGFBP7] values of $0.3(\mathrm{ng} / \mathrm{mL})^{2} / 1,000$ or greater identify patients at high risk and those $>2(\mathrm{ng} / \mathrm{mL})^{2} / 1,000$ at highest risk for $\mathrm{AKI}$, and provide new information to support the clinical decision-making. For post-operative surgical ICU patients, the urinary [TIMP-2] [IGFBP7] test accurately identified patients at risk for developing AKI within the ensuing 12 hours, and its inclusion in the clinical risk prediction models significantly enhanced their performance.

\section{THE ROLE OF BIOMARKERS IN EARLY DIAGNOSIS OF AKI}

Conventional diagnosis of AKI depends on oliguria and the rise of serum creatinine level, both of which can be delayed markers of kidney damage. Delayed diagnosis of AKI in the critically ill patients is related to increased morbidity and mortality, prolonged length of stay, and cost escalation. There are promising candidate biomarkers that reflect kidney and tubular function, detect an early and graded increase in tubular epithelial cell injury, and distinguish prerenal disease from acute tubular necrosis (ATN).

NGAL, detectable in the urine as early as 3 hours after renal injury, shows promise as a biomarker to help diagnose early AKI. NGAL has been tested in multiple studies of patients at risk for AKI due to sepsis, cardiac surgery, exposure to contrast media, or after renal transplantation. In these studies, the average sensitivity and specificity of NGAL measured one to three days prior to AKI diagnosis was 76 and 77 percent respectively, for cardiac surgery patients, and 73 and 80 percent respectively, for patients admitted to the ICU. ${ }^{[5]}$

In a prospective study of patients undergoing cardiac surgery, each of the biomarkers urine cystatin C, NGAL, KIM-1, alpha glutathione S-transferases ( $\alpha$-GST), and piGST demonstrated the ability to diagnose stage 3 AKI at various postoperative time points. ${ }^{[6]}$ Preoperative KIM-1 and alpha-GST predicted the development of stage 1 and stage 3 AKI. KIM-1 was very useful in differentiating ATN from other forms of AKI.

[IGFBP7] [TIMP-2] biomarker panel best predicted AKI at 12 hours with area under the receiver operating characteristics curve (AUCs) of 0.77 and 0.75 , respectively. These markers performed well in patients with sepsis (AUC 0.82 ) and post-operatively (AUC 0.85) when compared to traditional biomarkers, and improved risk stratification for AKI well ahead of clinical manifestations (azotemia and oliguria).

Urinary excretion of urinary liver-type fatty acid-binding protein (L-FABP) reflects stress of proximal tubular epithelial cells and correlates with severity of ischemic tubular injury. A meta-analysis of 15 prospective cohort studies demonstrated that L-FABP can discriminate AKI in hospital-based cohorts of high-risk patients.

Other potential biomarkers for diagnosis of AKI include proteomics, microRNAs, urinary tubular enzymes, and urinary low-molecular-weight proteins. Further studies are needed to clarify the role of these markers.

Biomarkers in conjunction with clinical judgment may be ideal for determining the likelihood that a patient will develop AKI in the next 24 hours. A clinical model of renal angina index (RAI) has been developed to identify which critically ill patients would be at the greatest risk of development of AKI. ${ }^{[7,8]}$ Renal angina is a model that uses patient demographic risk factors and clinical context in addition to early signs of injury such as oliguria or fluid overload to develop a practice to identify patients 
at high risk for AKI. The identification should heighten the vigilance in monitoring of kidney function and give a signal to the care providers to use tactics to improve renal outcomes by increasing kidney perfusion and avoiding nephrotoxins. ${ }^{[0]}$ RAI has high negative predictive value to rule out AKI. When biomarkers were combined with the RAI, either individually or in pairs, the predictive performance for diagnosis of AKI improved significantly (AUC 0.80 RAI alone, increased to 0.84-0.88; $P<0.05$ for each). In addition, the AUC for a single measurement of [IGFBP7] [ [TIMP-2] to predict AKI at 12 hours was 0.82 (95\% CI: 0.76-0.88) for biomarkers alone and 0.86 (95\% CI: 0.80-0.90) when biomarkers were combined with clinical variables. ${ }^{[10]}$ The furosemide stress test (FST) can be used for the purpose of evaluating tubular integrity and nephron function in the absence of a kidney biopsy. This combined serial testing, including initial testing with a strong negative predictive value (e.g., renal angina, biomarkers, etc.) followed by a more selective testing technique with higher positive predictive values (e.g., biomarkers, FST, etc.) could improve the diagnostic value for AKI. ${ }^{[1]}$

\section{THE ROLE OF BIOMARKERS IN AKI INTERVENTION}

Biomarkers may be useful in selecting patients who might benefit from various treatment modalities for AKI. Clinicians can use these biomarkers to determine which patients might be candidates for novel therapeutic strategies intended to improve kidney outcomes. Remote ischemic preconditioning (RIPC) with upper-arm blood pressure cuff inflations prior to surgery was used to prevent or decrease ischemic reperfusion induced AKI in patients for cardiac surgery. In those who had an increase in [TIMP-2]·[IGFBP7] after RIPC and prior to cardiopulmonary bypass, the incidence of AKI was reduced compared to those who did not have elevated cell cycle arrest biomarkers. ${ }^{[12]}$ This suggests that increases in biomarkers may be used to predict which patients will respond to RIPC, a potential therapeutic intervention for the prevention of AKI. The important differences that exist between biomarker-positive versus biomarkernegative individuals may dictate a predicted response to the therapy. ${ }^{[13]}$ The measurement of urinary angiotensinogen could play a role in identifying patients with AKI who are likely to develop CKD, and could potentially benefit from the renin-angiotensin system (RAS) blockade. ${ }^{[14,15]}$

Biomarkers may also be able to guide the decision making for the initiation of renal replacement therapy (RRT) in patients with AKI. Selected studies demonstrated that NGAL, cystatin-C, NAG, KIM-1, and a1-microglobulin had the potential to distinguish patients in whom RRT will be needed. This would imply that these biomarkers may be integrated into clinical decision algorithms, and could synergistically improve current ability to initiate RRT early. ${ }^{[16,17]}$ However, published studies have many recognized limitations, which preclude the ability to adapt their findings into clinical practice today. While the currently available data are not sufficient to conclude that biomarkers should be used routinely for clinical decision making for RRT initiation, additional data may in the future significantly modify the clinical variability for initiation of RRT, and potentially translate into improved outcomes and costeffectiveness. A potential approach to future biomarker strategies for RRT initiation, integrating these biomarkers with traditional clinical factors, has been proposed.

Biomarkers could be used to exclude those participants who are at low risk for an outcome, to enrich the study population sampled, and therefore increase the event rate during clinical trials. Using biomarkers to aid in the selection of a higher-risk patient group for a study could lead to a focus on those more likely to benefit from an intervention. ${ }^{[18,19]}$ In the recent ELAIN trial, KDIGO classification combined with plasma NGAL (>150 ng/dL) was used to randomize patients for early versus late RRT. Similarly, the use of enrichment is also important clinically since efforts to improve clinical outcomes will be most effective when focused on the highest-risk groups. ${ }^{[20]}$

\section{THE ROLE OF BIOMARKERS IN AKI PROGNOSIS}

The ability of biomarkers to predict AKI progression to chronic kidney disease (CKD) or renal recovery has been investigated. Proteinuria and microalbuminuria, the classical markers of CKD progression, have been used and validated for the progression of AKI to CKD. KIM-1, NGAL, and urinary cystatin $C$ could play a role in the prediction of renal recovery. The role of biomarkers including NGAL, KIM1 , and nephronectin (NPNT) in the recovery process has been studied, but it has not reached the point of widespread clinical implementation. The activation of RAS has long been recognized as an important contributor to chronic renal injury. Urinary angiotensinogen has been proposed as a marker of intrarenal RAS activity, and it is predictive of progression of CKD. Information regarding specific biomarkers of renal repair or progression is scarce but growing.

Many studies have established an association between AKI biomarkers and short-term outcomes defined by need for RRT, prolonged hospitalizations, and death. The TRIBE investigators have demonstrated that the highest tertile of peak urinary NGAL, IL-18, KIM-1, LFABP, and albumin was independently associated with a 2.0- to 3.2-fold increased risk for mortality compared with the 
lowest biomarker tertile. Preoperative and postoperative plasma NGAL were associated with long-term outcomes such as 3-year mortality rates. A [TIMP-2] - [IGFBP7] value greater than $2(\mathrm{ng} / \mathrm{mL})^{2} / 1,000$ was associated with increased mortality or a need for renal replacement therapy over the next 9 months. Measurement of urinary angiotensinogen may allow the prediction of severe AKI and other adverse outcomes in ICU. The combination of urinary angiotensinogen and renin predicts the progression to very severe disease in patients with early AKI after cardiac surgery. NGAL was useful for predicting renal recovery, defined as being alive, not requiring dialysis during hospitalization or having a persistent RIFLE-F classification at the time of hospital discharge.

In conclusion, traditional parameters provide some guidance for the diagnosis of AKI but early, more sensitive, affordable, specific, clinically acceptable, biomarkers of AKI are also needed. As the development of proteomics, metabolomics and basic biological understanding of AKI progresses, more and more novel biomarkers will be identified. The combination of biomarkers indicating kidney dysfunction and damage is likely to have higher sensitivity and specificity. Although many candidate biomarkers have been identified, the validation in different settings of AKI, the development and testing of rapid assays and the development of a panel of biomarkers are necessary before they are used clinically.

\section{Source of Foundation}

This study was partially supported by the National Natural Science Foundation of China (81560131).

\section{Conflict of Interest}

None declared.

\section{REFERENCES}

1. Lee SH, Youn YN, Choo HC, Lee S, Yoo KJ. Cystatin C as a predictive marker of renal dysfunction and mid-term outcomes following off-pump coronary artery bypass grafting. Heart 2015;101:1562-8.

2. McIlroy DR, Farkas D, Matto M, Lee HT. Neutrophil gelatinase-associated lipocalin combined with delta serum creatinine provides early risk stratification for adverse outcomes after cardiac surgery: a prospective observational study. Crit Care Med 2015; 43:1043-52.

3. Chawla LS, Abell L, Mazhari R, Egan M, Kadambi N, Burke HB, et al. Identifying critically ill patients at high risk for developing acute renal failure: A pilot study. Kidney Int 2005;68:2274-80.
4. Kashani K, Al-Khafaji A, Ardiles T, Artigas A, Bagshaw SM, Bell M, et al. Discovery and validation of cell cycle arrest biomarkers in human acute kidney injury. Crit Care 2013; 17:R25.

5. Yang $\mathrm{CH}$, Chang $\mathrm{CH}$, Chen TH, Fan PC, Chang SW, Chen CC, et al. Combination of Urinary Biomarkers Improves Early Detection of Acute Kidney Injury in Patients With Heart Failure. Circ J 2016;80:1017-23.

6. Takaya Y, Yoshihara F, Yokoyama H, Kanzaki H, Kitakaze M, Goto Y, et al. Risk Stratification of Acute Kidney Injury Using the Blood Urea Nitrogen/Creatinine Ratio in Patients With Acute Decompensated Heart Failure. Circ J 2015;79:1520-5.

7. Schley G, Koberle C, Manuilova E, Rutz S, Forster C, Weyand M, et al. Comparison of Plasma and Urine Biomarker Performance in Acute Kidney Injury. PloS one 2015; 10:e0145042.

8. Cruz DN, de Geus HR, Bagshaw SM. Biomarker strategies to predict need for renal replacement therapy in acute kidney injury. Semin Dial 2011;24:124-31.

9. Fuhrman DY, Kellum JA. Biomarkers for Diagnosis, Prognosis and Intervention in Acute Kidney Injury. Contrib Nephrol 2016;187:47-54.

10. Bihorac A, Chawla LS, Shaw AD, Al-Khafaji A, Davison DL, Demuth GE, et al. Validation of cell-cycle arrest biomarkers for acute kidney injury using clinical adjudication. Am J Respir Crit Care Med 2014;15;189:9329.

11. Murray PT, Mehta RL, Shaw A, Ronco C, Endre Z, Kellum JA, et al. Potential use of biomarkers in acute kidney injury: report and summary of recommendations from the 10th Acute Dialysis Quality Initiative consensus conference. Kidney Int 2014;85:513-21.

12. Meersch M, Schmidt C, Van Aken H, Martens S, Rossaint J, Singbartl K, et al. Urinary TIMP-2 and IGFBP7 as early biomarkers of acute kidney injury and renal recovery following cardiac surgery. PLoS One 2014; 27; 9:e93460.

13. McMahon BA, Koyner JL. Risk Stratification for Acute Kidney Injury: Are Biomarkers Enough? Adv Chronic Kidney Dis 2016;23:167-78.

14. Kashani K, Kellum JA. Novel biomarkers indicating repair or progression after acute kidney injury. Curr Opin Nephrol Hypertens 2015;24:21-7.

15. Koyner JL, Shaw AD, Chawla LS, Hoste EA, Bihorac A, Kashani K, et al. Tissue Inhibitor Metalloproteinase-2 (TIMP-2).IGF-Binding Protein-7 (IGFBP7) Levels Are Associated with Adverse Long-Term Outcomes in Patients with AKI. J Am Soc Nephrol 2015;26:1747-54.

16. Cooper DS, Claes D, Goldstein SL, Bennett MR, Ma Q, Devarajan P, et al. Follow-Up Renal Assessment of Injury Long-Term After Acute Kidney Injury (FRAIL-AKI). Clin J Am Soc Nephrol2016; 11:21-9.

17. Basu RK, Wong HR, Krawczeski CD, Wheeler DS, Manning PB, Chawla LS, et al. Combining Functional and Tubular Damage Biomarkers Improves Diagnostic Precision for Acute Kidney Injury after Cardiac Surgery. J Am Coll Cardiol 2014;64:2753-62.

18. Alge JL, Arthur JM. Biomarkers of AKI: a review of mechanistic relevance and potential therapeutic implications. Clin J Am Soc Nephrol 2015;7;10:147-55.

19. Basu RK, Kaddourah A, Terrell T, Mottes T, Arnold P, Jacobs J, et al. Assessment of Worldwide Acute Kidney Injury, Renal Angina and Epidemiology in Critically Ill Children (AWARE): A Prospective Study to Improve Diagnostic Precision. J Clin Trials 2015;5:1-17.

20. Zarbock A, Kellum JA, Schmidt C, Van Aken H, Wempe C, Pavenstädt $\mathrm{H}$, et al. Effect of Early vs. Delayed Initiation of Renal Replacement Therapy on Mortality in Critically Ill Patients With Acute Kidney Injury: The ELAIN Randomized Clinical Trial. JAMA 2016;315:2190-9.

How to cite this article: Peng ZY. The biomarkers for acute kidney injury: A clear road ahead? J Transl Intern Med 2016; 4: 95-8. 